María Atilia Gómez, ${ }^{1,2}$ Ph.D.; María Serena Peñaherrera, ${ }^{2}$ Ph.D.; Víctor Aguirre-Tello, ${ }^{2}$; Margarita Vela-Cavinato, ${ }^{2}$; and Guillermo Giovambattista, ${ }^{1}$ Ph.D.

\title{
Allele Frequencies of 15 STR Loci in the Population of the City of Quito, Ecuador
}

POPULATION: Quito City Population (Ecuador, South America, $n=116-207)$.

KEYWORDS: forensic science, DNA typing, Quito population, short tandem repeats, population genetics, D3S1358, THO1, D21S11, D18S51, PENTA E, D5S818, D13S317, D7S820, D16S539, CSF1PO, PENTAD, VWA, D8S1179, TPOX, FGA

Whole blood obtained by venipuncture was collected from unrelated individuals residing in Quito City, Ecuador, South America, using one 1.2-mm micropunch of bloodstains collected on FTA Classic Cards (Whatman FTA ${ }^{\circledR}$ ), and the DNA was extracted following the manufacturer's directions. PCR amplification of the 15 STR loci (D3S1358, THO1, D21S11, D18S51, PENTA E, D5S818, D13S317, D7S820, D16S539, CSF1PO, PENTAD, VWA, D8S1179, TPOX, FGA) was performed using the PowerPlex ${ }^{\circledR} 16$ System kit (Promega Corp., Madison, WI) (1) (PE-Biosystems, Foster City, CA) following the manufacturer's protocol. The amplified products were separated and detected using the ABI Prism ${ }^{\circledR}$ 310 genetic analyzer (Applied Biosystems, Foster City, CA) using the ABI Prism GeneScan analysis software version 3.1 and Genotyper DNA fragment analysis software version 2.5.2 (2).

The data were analyzed using Power-Stats (3). Allele frequencies and other relevant information from analysis of the 15 STR loci are given in Table 1 . The complete dataset is available to any interested researcher upon request, or can be downloaded from the following World Wide Web site: http://www.fcv.unlp.edu.ar/ info-general/publicaciones/pdf/supplemtary.pdf.

\section{Acknowledgment}

This work was supported by the Cruz Roja Ecuatoriana (CRE).

\section{References}

1. Promega Corporation. PowerPlex ${ }^{\circledR} 16$ system, technical manual part TMD012, revised edn. Madison, WI: Promega Corporation, March 2001.

2. PE Applied Biosystems. ABI PRISM ${ }^{\circledR} 310$ Genetic Analyzer user's manual. Perkin Foster City, CA: Elmer Corporation, 1998.

3. Teraba A. Tools for analysis of population statistics. Prof DNA 1999;2(3).

Additional information and reprint requests:

María Atilia Gómez, Ph.D.

Centro de Investigaciones en Genética Básica y Aplicada

Facultad de Ciencias Veterinarias

Universidad Nacional de La Plata

La Plata B1900AVW, cc 296

Argentina

E-mail: matilia@arnet.com.ar
${ }^{1}$ Centro de Investigaciones en Genética Básica y Aplicada, Facultad de Ciencias Veterinarias, Universidad Nacional de La Plata, La Plata B1900AVW, cc 296, Argentina.

${ }^{2}$ Cruz Roja Ecuatoriana (CRE), Laboratorio de Genética, Quito, Ecuador. 
TABLE 1-STR allele frequencies of the 15 loci in the population of the city of Quito (Ecuador).

\begin{tabular}{|c|c|c|c|c|c|c|c|c|c|c|c|c|c|c|c|}
\hline Allele & D3S1358 & THO1 & D21S11 & D18S51 & PENTA E & D5S818 & D13S317 & D7S820 & D16S539 & CSF1PO & PENTAD & VWA & D8S1179 & TPOX & FGA \\
\hline 5 & - & - & - & - & $2.3 \%$ & - & - & - & - & - & - & - & - & - & - \\
\hline 6 & - & $36.1 \%$ & - & - & - & $0.3 \%$ & - & - & - & - & - & - & - & - & - \\
\hline 7 & - & $33.2 \%$ & - & - & $5.6 \%$ & $10.3 \%$ & - & $0.3 \%$ & - & - & $0.6 \%$ & - & - & - & - \\
\hline 8 & - & $7.7 \%$ & - & - & $1.0 \%$ & $0.7 \%$ & $8.2 \%$ & $4.4 \%$ & $1.3 \%$ & $0.7 \%$ & $1.6 \%$ & - & - & $50.0 \%$ & - \\
\hline 9 & - & $7.4 \%$ & - & - & $0.3 \%$ & $6.0 \%$ & $25.2 \%$ & $4.0 \%$ & $20.5 \%$ & $1.0 \%$ & $21.1 \%$ & - & $0.7 \% 9$ & $4.3 \%$ & - \\
\hline 9.3 & & $14.8 \%$ & - & - & - & - & - & - & - & - & - & - & - & - & - \\
\hline 10 & - & $0.6 \%$ & - & $2.2 \%$ & $2.3 \%$ & $4.3 \%$ & $9.9 \%$ & $23.8 \%$ & $20.1 \%$ & $24.3 \%$ & $27.3 \%$ & - & $7.2 \%$ & $2.6 \%$ & - \\
\hline 11 & - & - & - & $2.9 \%$ & $6.3 \%$ & $50.7 \%$ & $14.9 \%$ & $38.6 \%$ & $25.2 \%$ & $28.8 \%$ & $11.4 \%$ & - & $6.5 \%$ & $27.6 \%$ & - \\
\hline 12 & $0.6 \%$ & - & - & $8.4 \%$ & $18.4 \%$ & - & $21.3 \%$ & $25.8 \%$ & $21.8 \%$ & $35.8 \%$ & $16.2 \%$ & - & $16.4 \%$ & $15.5 \%$ & - \\
\hline 13 & - & - & - & $9.5 \%$ & $4.9 \%$ & - & $14.9 \%$ & $3.0 \%$ & $8.7 \%$ & $8.3 \%$ & $14.3 \%$ & - & $33.3 \%$ & - & - \\
\hline 14 & $8.1 \%$ & - & - & $24.8 \%$ & $3.9 \%$ & - & $5.3 \%$ & - & $2.3 \%$ & $1.0 \%$ & $5.8 \%$ & $3.6 \%$ & $20.3 \%$ & - & - \\
\hline 15 & $47.4 \%$ & - & - & $13.9 \%$ & $16.4 \%$ & - & $0.4 \%$ & - & - & - & $1.3 \%$ & $7.5 \%$ & $11.8 \%$ & - & - \\
\hline 16 & $26.3 \%$ & - & - & $14.6 \%$ & $7.9 \%$ & - & - & - & - & - & $0.3 \%$ & $37.7 \%$ & $3.6 \%$ & - & - \\
\hline 17 & $12.7 \%$ & - & - & $12.4 \%$ & $6.3 \%$ & - & - & - & - & - & - & $34.4 \%$ & - & - & $1.3 \%$ \\
\hline 18 & $4.9 \%$ & - & - & $6.6 \%$ & $7.9 \%$ & - & - & - & - & - & - & $13.3 \%$ & - & - & $0.9 \%$ \\
\hline 18.2 & - & - & - & - & - & - & - & - & - & - & - & - & - & - & - \\
\hline 19 & - & - & - & $2.2 \%$ & $3.9 \%$ & - & - & - & - & - & - & $2.6 \%$ & - & - & $9.9 \%$ \\
\hline 20 & - & - & - & $1.1 \%$ & $6.6 \%$ & - & - & - & - & - & - & $1.0 \%$ & - & - & $6.9 \%$ \\
\hline 21 & - & - & - & $0.4 \%$ & $3.0 \%$ & - & - & - & - & - & - & - & - & - & $9.1 \%$ \\
\hline 22 & - & - & - & $0.4 \%$ & $3.0 \%$ & - & - & - & - & - & - & - & - & - & $12.1 \%$ \\
\hline 23 & - & - & - & $0.4 \%$ & - & - & - & - & - & - & - & - & - & - & $13.8 \%$ \\
\hline 24 & - & - & - & $0.4 \%$ & - & - & - & - & - & - & - & - & - & - & $14.7 \%$ \\
\hline 25 & - & - & - & - & - & - & - & - & - & - & - & - & - & - & $15.5 \%$ \\
\hline 26 & - & - & - & - & - & - & - & - & - & - & - & - & - & - & $10.3 \%$ \\
\hline 27 & - & - & $1.70 \%$ & - & - & - & - & - & - & - & - & - & - & - & $2.6 \%$ \\
\hline 28 & - & - & $7.50 \%$ & - & - & - & - & - & - & - & - & - & - & - & $2.2 \%$ \\
\hline 29 & - & - & $20.80 \%$ & - & - & - & - & - & - & - & - & - & - & - & $0.9 \%$ \\
\hline 29.2 & - & - & $0.80 \%$ & - & - & - & - & - & - & - & - & - & - & - & - \\
\hline 30 & - & - & $17.50 \%$ & - & - & - & - & - & - & - & - & - & - & - & - \\
\hline 30.2 & - & - & $1.70 \%$ & - & - & - & - & - & - & - & - & - & - & - & - \\
\hline 31 & - & - & $11.70 \%$ & - & - & - & - & - & - & - & - & - & - & - & - \\
\hline 31.2 & - & - & $18.30 \%$ & - & - & - & - & - & - & - & - & - & - & - & - \\
\hline 32 & - & - & $0.80 \%$ & - & - & - & - & - & - & - & - & - & - & - & - \\
\hline 32.2 & - & - & $14.20 \%$ & - & - & - & - & - & - & - & - & - & - & - & - \\
\hline 33.2 & - & - & $5 \%$ & - & - & - & - & - & - & - & - & - & - & - & - \\
\hline$n$ & 154 & 155 & 119 & 137 & 152 & 151 & 141 & 149 & 149 & 144 & 154 & 154 & 207 & 152 & 116 \\
\hline$h_{\mathrm{o}}$ & 62.30 & 71.00 & 81.50 & 81.00 & 86.80 & 65.60 & 87.20 & 77.50 & 77.2 & 71.50 & 79.90 & 69.50 & 79.70 & 63.80 & 86.20 \\
\hline$h_{\mathrm{e}}$ & 68.33 & 72.79 & 84.40 & 85.09 & 90.58 & 68.46 & 83.06 & 72.55 & 80.35 & 72.53 & 82.02 & 71.67 & 80.74 & 64.93 & 89.08 \\
\hline PD & 0.852 & 0.877 & 0.9491 & 0.960 & 0.977 & 0.859 & 0.943 & 0.872 & 0.931 & 0.870 & 0.938 & 0.873 & 0.930 & 0.821 & 0.9686 \\
\hline $\mathrm{PE}$ & 0.320 & 0.443 & 0.6274 & 0.618 & 0.731 & 0.363 & 0.739 & 0.468 & 0.548 & 0.452 & 0.597 & 0.420 & 0.594 & 0.339 & 0.7188 \\
\hline
\end{tabular}

- , allele not detected or not applicable; $n$, sample size; $h_{\mathrm{o}}$, observed heterozygosity $(\%) ; h_{\mathrm{e}}$, expected heterozygosity (\%); PD, power of discrimination; PE, power of exclusion. 